\title{
Uric acid nephrolithias in the era of noncontrast computed tomography
}

\author{
Blayne K. Welk, MD; Joel M.H. Teichman, MD, FRCSC
}

\begin{abstract}
We report 2 patients who presented with uric acid renal calculi. Both patients had previously been treated unsuccessfully for their stones with shock wave lithotripsy and were referred to our centre for percutaneous nephrolithomy. We were able to avoid surgery in both cases owing to the recognition of uric acid stone composition despite the calculi being visible on CT scout films.
\end{abstract}

CUAJ 2008;2(4):420-1

\section{Introduction}

Noncontrast CT scanning has become the standard imaging test for patients with renal colic. CT detects almost all stone types and has the added advantage of diagnosing other intra-abdominal pathology. Before the widespread use of CT, uric acid stones were often suspected when an intravenous pyelogram suggested obstruction either with a smoothcontoured filling defect or without an associated radiopaque stone. The widespread use of CT has largely replaced plain film radiography in the investigation of acute renal colic. We have observed physicians using the digital scout film from the CT as a replacement for an abdominal plain film and assuming that the same generalizations for radiopaque calculi apply. We describe 2 cases of large uric acid stones that were radiopaque on CT digital scout film and successfully treated with medical therapy.

\section{Case presentation and management}

Patient 1 was a 79-year-old woman with a history of shock wave lithotripsy used to treat recurrent renal calculi. Her medical history included diabetes mellitus, hypertension and atrial fibrillation requiring anticoagulation. CT scan demonstrated a 2.9-cm renal pelvis calculus, which was visible on the digital CT scout film (Fig. 1). The mean stone attenuation was 337 Hounsfield units (HU) from the cross-sectional imaging, which suggested the presence of a uric acid stone.

The second patient was an 89-year-old man referred after failing shock wave lithotripsy (the operative report by the urologist from the prior shock wave lithotripsy commented that the stones were not visualized on fluoroscopy). His medical history was significant for a cardiac pacemaker and atrial fibrillation requiring anticoagulation. His CT scan showed a partial left staghorn calculus and a $1.6-\mathrm{cm}$ right renal stone (mean stone attenuation of $320 \mathrm{HU}$ ); the left partial staghorn was also visualized on the digital scout film (Fig. 2).
Owing to these patients' ages and comorbidities, a nonsurgical approach was particularly desirable. Medical dissolution therapy (consisting of potassium citrate $25 \mathrm{mEq} 3$ times daily) was started in both patients. Both patients were reassessed after 6 weeks with a repeat CT scan of the kidneys, ureter and bladder, which demonstrated a decrease in stone size. Patient 1 was switched to a sodium citrate solution $(30 \mathrm{~mL} / \mathrm{d})$ owing to hyperkalemia. Both patients were stone-free within 7 months. Patient 1 had 2 renal calculi recurrences (consistent with uric acid stones) that were successfully managed with dissolution therapy.

\section{Discussion}

The digitally rendered scout film generated by the CT scan is often used as a substitute for a plain film of the abdomen. Plain film radiography and the digital scout $\mathrm{CT}$ do not appear to be interchangeable in their ability to detect urinary tract calculi. ${ }^{1,2,3}$

Pure uric acid stones represent about $10 \%$ of calculus disease in elderly patients. ${ }^{4}$ Their etiology is a combination of low urinary output, hyperuricosuria and acidic urine $(\mathrm{pH}<5.0)$. A history of gout or diabetes should also alert the clinician to the possibility of uric acid stones. Pure uric acid stones are generally not visible on plain radiographs. Uric acid stones may be suspected on CT scan based on a stone attenuation of 200-600 HU. ${ }^{5}$ Specific issues in the work up of a patient with suspected uric acid stones include a history of gout, high fluid and protein intake, and previous difficulties visualizing the stone. Investigations should include serum urate, urinary analysis for $\mathrm{pH}$ and imaging studies. For large stones, a CT scan of the kidneys, ureter and 


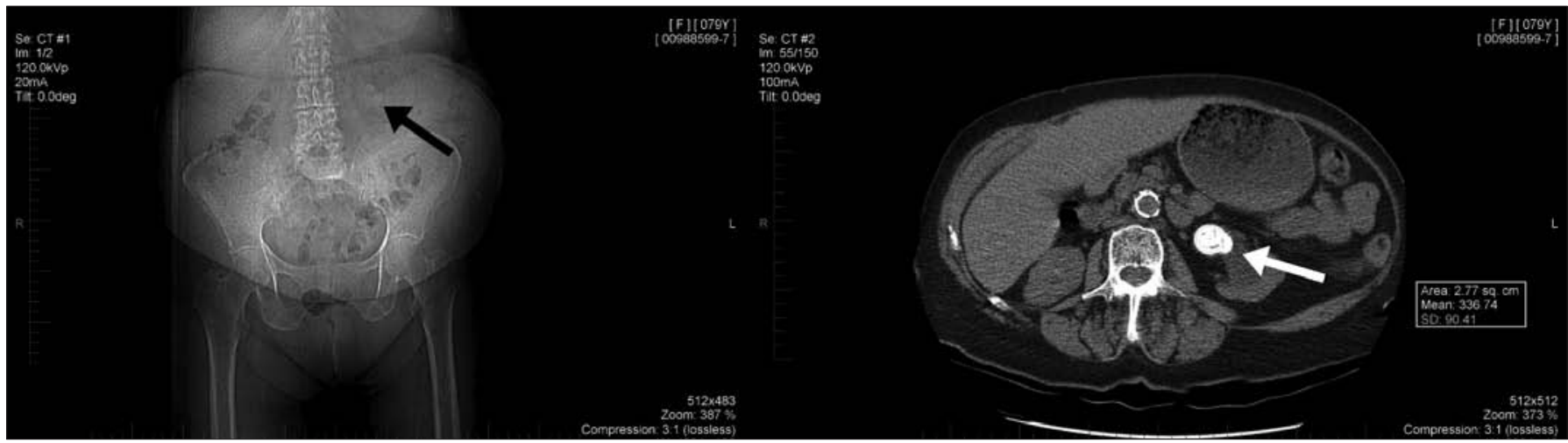

Fig. 1. Digital scout film (left) and CT scan (right) of a 79-year-old woman showing a 2.9-cm renal pelvis stone (arrows). Mean Hounsfield units of 337 were measured on CT (abdominal windows).

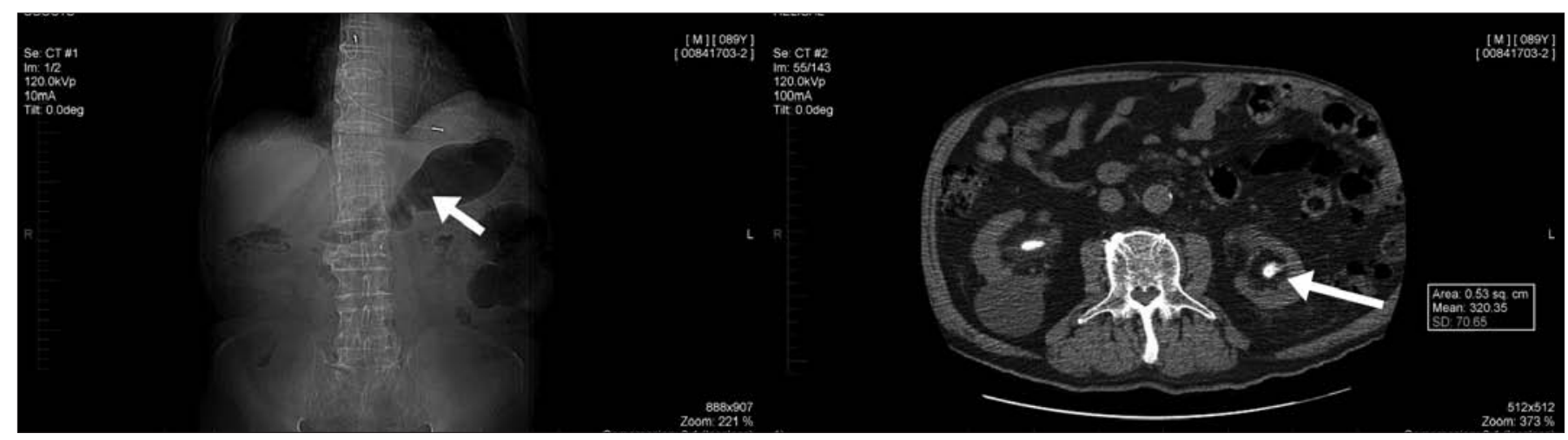

Fig. 2. Digital scout film (left) and CT scan (right) of an 89-year-old man showing a 1.6-cm right renal pelvis stone and a left partial staghorn calculus. The left calculus is also visible on the digital scout film (arrow). Mean Hounsfield units of 320 were measured on CT (abdominal windows).

bladder may be sufficient if a Hounsfield attenuation can be measured without the confounding factor of volume averaging, as may occur in smaller stones where adjacent soft tissue is included in the area of interest. For smaller stones, plain radiography may be useful to confirm that the stone is radiolucent. Clearly, in patients with prior urolithiasis, stone composition analysis is informative.

These cases offer an important reminder in the era of CT scans to look for uric acid stones. Medical dissolution therapy of pure uric acid stones is successful in up to $80 \%$ of patients. ${ }^{4}$ The CT scout film is a digital film generated from the helical CT; the appearance of calculi on this film is dependent on the kilovolt and milliampere settings used, ${ }^{6}$ and radiopacity on this film does not necessarily preclude a uric acid stone.

From the Division of Urology, Providence Healthcare, Vancouver, BC, and the Department of Urologic Sciences, University of British Columbia, Vancouver, BC
This article has been peer reviewed.

Competing interests: None declared.

\section{References}

1. Assi Z, Platt JF, Francis IR, et al. Sensitivity of CT scout radiography and abdominal radiography for revealing ureteral calculi on helical CT. AJR Am J Roentgenol 2000;175: 333-7.

2. Ege G, Akman H, Kuzucu K, et al. Can computed tomography scout radiography replaced plain film in the evaluation of patients with acute urinary tract colic. Acta Radiol 2004;4:469-73.

3. Chu G, Rosenfield AT, Anderson K, et al. Sensitivity and value of digital CT radiography for detecting ureteral stones in patients with ureterolithiasis diagnosed on unenhanced CT. AJR Am J Roentgenol 1999;173:417-23.

4. Shekarriz, Stoller ML. Uric acid nephrolithiasis: current concepts and controversies. J Urol 2002;168:1307-14.

5. Sheir KZ, Mansour O, Madbouly K, et al. Determination of the chemical composition of urinary calculi by noncontrast computerized tompography. Urol Res 2005;33:99-104.

6. Tublin ME, Murphy ME, Delong DM, et al. Conspicuity of renal calculi at unenhanced CT: effects of calculus composition and size and CT technique. Radiology 2002;225:91-6.

Correspondence: Dr. Joel Teichman, Division of Urology, St. Paul's Hospital, Burrard Bldg. C307, 1081 Burrard St., Vancouver BC V6Z 1Y6 